\title{
Elemente und Ephemeride des Cometen 1898...(Chase).
}

Aus den Beobachtungen Cambridge Nov. 14 und Teramo Dec. 5 hat Herr $\mathcal{F}$. Möller mit möglichstem Anschluss an die Lick-Beobachtungen Nov. 23 und 25 dis folgenden parabolischen Elemente und Ephemeride abgeleitet.

$$
\begin{aligned}
& \omega=4^{\circ} 23^{\prime} 9^{\prime \prime} 2 \\
& \delta=\begin{array}{lll}
95 & 47 & 0.2
\end{array} \quad \text { I } 898.0 \\
& i=22 \quad 28 \quad 25.2
\end{aligned}
$$$$
T=1898 \text { Sept. I } 9.64919 \text { M. Z. Berlin }
$$

$\log q=0.357918$

Mittlere Oerter $(B-R)$ :

Nov. $23 \quad \Delta \lambda \cos \beta=-5.9 \quad \Delta \beta=+5.7$
Heliocentrische Aequatorealcoordinaten.

$$
\begin{aligned}
& x=[9.966075] r \cdot \sin \left(v+190^{\circ} 38^{\prime} 27^{\prime \prime} \cdot 5\right) \\
& y=[9.974571] r \cdot \sin \left(v+1085^{8} 29 \cdot 3\right) \\
& z=[9.703394] r \cdot \sin (v+560 \text { 18.0 }
\end{aligned}
$$

Correction der Ephemeride in A. N. 3530.

Dec. $22 \quad \Delta c=+1^{\mathrm{m}} 4^{\mathrm{s}} \quad \Delta \delta=-0: 8$
$=+141$

Ephemeride für $\mathbf{r}^{\text {h }}$ M. Z. Berlin.

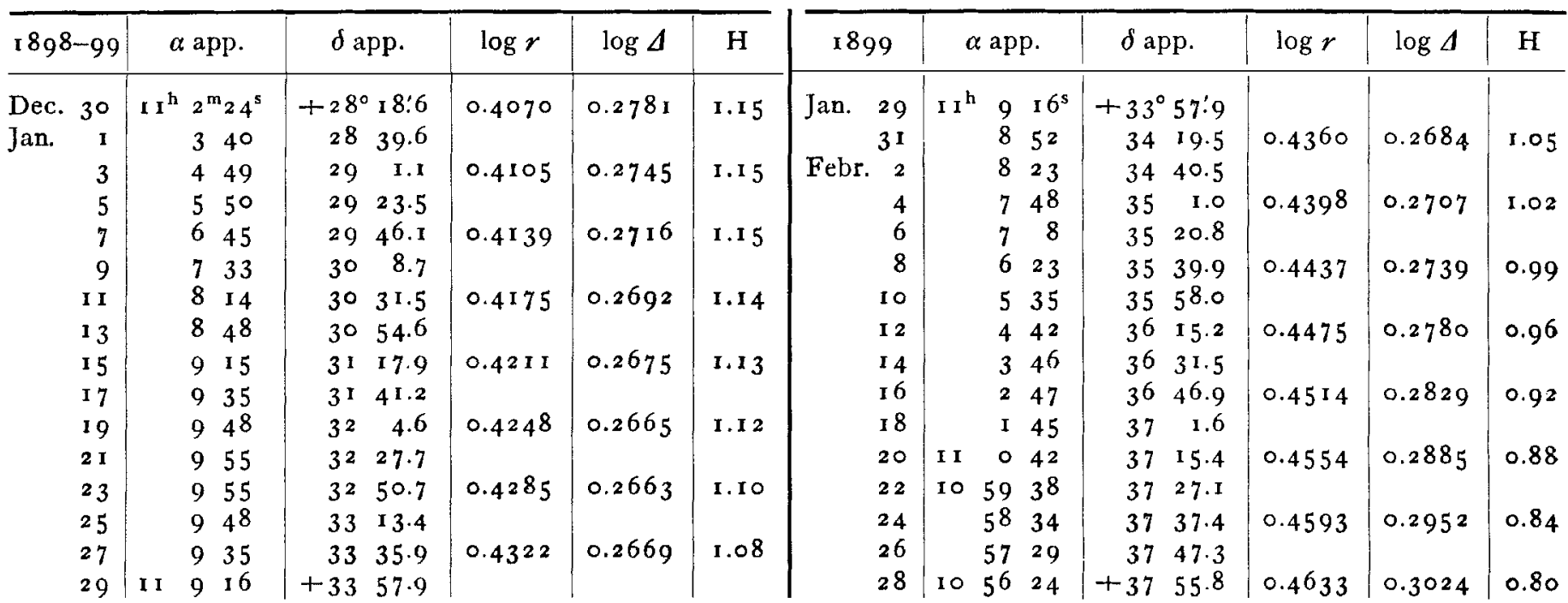

Ein Versuch aus Nov. 14, 25 und Dec. 5 einen Kegelschnitt ohne Voraussetzung einer Excentricität abzuleiten, ergab eine Hyperbel mit $e=1.025$. Dieselbe ist aber, da die Darstellung der Beobachtungen durch die Parabel noch eine genügende ist, zunächst nur als Rechnungsresultat anzusehen. Jedenfalls aber scheint hieraus hervorzugehen, dass der Comet keine Ellipse mit einer Umlaufszeit von 32 Jahren beschreibt, also auch nicht mit Comet 1867 I identisch sein kann. Gegen die Identität würde übrigens auch schon die mangelnde Uebereinstimmung der Elemente, die im Vergleich mit den früheren Elementen in A. N. $353^{\circ}$ noch schlechter geworden ist, sprechen.

Kiel 1898 Dec. 10.

H. Kreutz.

\section{Elemente des Cometen 1898... (Chase).}

Aus den Beobachtungen Cambridge Nov. 14, Mount Hamilton Nov. 24 und Teramo Dec. 5 habe ich die neben. stehenden parabolischen Elemente gerechnet.

Der mittlere Ort wird bis auf $+3 !^{\prime \prime} 3$ in Länge und $-0^{\prime \prime} 9$ in Breite dargestellt.

Wien 1898 Dec. 9 .

$$
\begin{aligned}
& T=1898 \text { Sept. } 2 \text { I.0015 M. Z. Berlin } \\
& \omega=4^{\circ} 59^{\circ} 4^{8} \text { 8.。 }^{\circ} \\
& \left.\delta=955^{8} 23.6\right\} 1898.0 \\
& i=223326.0 \\
& \log q=0.3^{60554}
\end{aligned}
$$

Berthold Cohn.

Planet (266) Aline, Corr. der Ephemeride (Veröff. R. I. Nr. 7): Dec. $6-1^{\mathrm{m}} \mathbf{1}^{\mathrm{s}}+\mathbf{1}^{\prime}$ 1. Gr. I r.1. E. Milloserich.

Planet (416) Vaticana. Corr. der Ephemeride (Veröff. R.I. Nr. 7): Dec. $5-27^{\text {s }}-2 \cdot 4$. Gr. 11.3. E. Millosevich.

Inhalt zu Nr. 353I. A. Müller. Aussergewöhnliche Meteorerscheinungen. 33. - W. F. Denning. Catalogue of i 77 long - cnduring and apparently stationary Radiant Points of Shooting Stars. 35. - A.W. Krassnow. Zur Integration der Jacobi'schen Differentialgleichung für die Mondbewegung. 37. - N. Herz. Zur Erklärung der Verdoppelung der Marscanäle. 4I. - Hammer. Württembergischbadischer Kartenanschluss. 43. - A. Abetti. Pianeta (397) (1894 BM). 43 - A. Schwassmann. Positionen von kleinen Planeten. 45. - M. Wolf. Mittheilung über Planetoiden. 45. - Aufforderung betr. Beobachtungen des Planeten (433) (1898 DQ) 45. - H. Kreuts. Elemente und Ephemeride des Cometen I898...(Chase). 47. - B. Cohn. Elemente des Cometen $1898 \ldots$ (Chase). 47. - E. Millosevich. Correctionen von Planeten-Ephemeriden. 47. 